\title{
Themenheft: Hydrogeologie in Österreich
}

\author{
Steffen Birk • Thilo Hofmann
}

Eingang des Beitrages: 13. 12.2009 / Eingang des überarbeiteten Beitrages: 14. 12.2009 / Online veröffentlicht: 27. 1.2010

(C) Springer-Verlag 2010

\section{Liebe Leserinnen und Leser,}

die nächste Tagung der FH-DGG wird im Mai 2010 in Tübingen als Drei-Länder-Tagung Deutschland - Österreich Schweiz stattfinden. Aus diesem Anlass möchten wir Ihnen in diesem Themenheft ausgewählte Fachbeiträge vorstellen, die in den Vortragsreihen „Hydrogeologie“ und „Umweltgeologie" bei der österreichischen Tagung PANGEO 2008 präsentiert wurden. Es versteht sich von selbst, dass eine solche Auswahl weder repräsentativ noch umfassend sein kann. Dennoch waren wir bestrebt, Ihnen ein möglichst breites Spektrum der in Österreich relevanten Themenbereiche zu präsentieren.

Österreich ist ein wasserreiches Land, welches mit Ausnahme der östlichsten Region im Bereich des Marchfeldes, des Neusiedler Sees und der Südoststeiermark über Wasserknappheit nicht klagen kann. Die Wasserversorgung beruht in Österreich in noch stärkerem Maße als in Deutschland auf der Nutzung von Grundwasserressourcen. Nach Angaben des Lebensministeriums bezieht ungefähr die eine Hälfte der Bevölkerung ihr Trinkwasser aus Quellwässern, die andere aus Grundwasservorkommen der quartären Tal- und Beckenfüllungen.

Insbesondere in den Tallagen bestehen Nutzungskonflikte, die oft jenen in Deutschland ähneln (Landwirtschaft, Industrie etc.). Aus der starken Beanspruchung alpiner Wasserressourcen ergeben sich zudem spezifische hydrogeolo-

Prof. Dr. S. Birk $(\bowtie)$

Institut für Erdwissenschaften, Karl-Franzens-Universität Graz, Heinrichstraße 26, 8010 Graz, Österreich

E-Mail: steffen.birk@uni-graz.at

Prof. Dr. T. Hofmann

Head Department of Environmental Geosciences, Universität

Wien, Althanstrasse 14, 1090 Wien, Österreich

E-Mail: thilo.hofmann@univie.ac.at gische Fragestellungen, beispielsweise im Zusammenhang mit Energiewirtschaft (Wasserkraft), Baumaßnahmen (Tunnelbau, Kraftwerksbau) und Tourismus (Beschneiung, Abwasser). Die Fachbeiträge in diesem Heft spiegeln die sich hieraus ergebende Themenvielfalt wider.

Achten \& Hofmann befassen sich mit der Charakterisierung von polyzyklischen aromatischen Kohlenwasserstoffen (PAK) aus Steinkohlen. Dabei werden unter anderem $\mathrm{Zu}$ sammenhänge zwischen dem Reifegrad der Kohlen und den PAK-Gehalten bzw. Mustern der Einzelsubstanzen aufgezeigt sowie umweltrelevante Aspekte wie Desorptionseigenschaften und Bioverfügbarkeit angesprochen.

Walther et al. stellen ein neuartiges Konzept zur Probennahme vor, das tiefenspezifisch und zeitlich hochaufgelöste hydrogeochemische Daten im Übergangsbereich zwischen ungesättigter und gesättigter Zone liefert. Damit wird eine wesentliche Grundlage für ein quantitatives Verständnis von Strömungs- und Transportprozessen in diesem Bereich geschaffen, welches etwa im Zusammenhang mit der Bewirtschaftung von Porengrundwasservorkommen in intensiv landwirtschaftlich genutzten Tallagen von hoher Relevanz ist.

Der Beitrag von Schubert et al. befasst sich mit hydrogeochemischen Besonderheiten in der böhmischen Masse. Die Grundwässer im dortigen Untersuchungsgebiet zeigen hohe Gehalte an Radon-222, die signifikant mit dem Urangehalt der kristallinen Festgesteine korrelieren und daher wertvolle Hinweise für die Erstellung von Radonpotenzialkarten liefern können.

Benischke et al. erarbeiten anhand des Fallbeispiels Kaisergebirge (Tirol) einen methodischen Ansatz zur Erkundung von Karstgrundwasserleitern in Gebirgsräumen. Durch den Einsatz flächendetaillierter hydrologischer Modellierung und Einbeziehung von Isotopendaten, Ergebnissen von Markierungsversuchen und geologisch-tektonischer Kennt- 
nisse werden die unterirdischen Entwässerungsrichtungen bestimmt und ein wichtiger Beitrag zur Abgrenzung der hydrographischen Einzugsgebiete geleistet.

Winkler et al. untersuchen die hydraulische Wirkung von Störungskernzonen im kristallinen Festgestein des Semmering-Rax-Gebiets. Am Beispiel eines obertägigen Aufschlusses der Talhofstörung wird die hydraulische Durchlässigkeit in drei Raumrichtungen quantifiziert und mit Ergebnissen von Packertests in derselben tektonischen Einheit verglichen. Derartige Untersuchungen liefern we- sentliche Kenntnisse beispielsweise für Prognosen von Wassereinbrüchen beim Tunnelvortrieb.

Wir hoffen, dieses Themenheft ermöglicht Ihnen interessante Einblicke in hydrogeologische Untersuchungen innerhalb Österreichs und freuen uns auf spannende Fachdiskussionen bei der kommenden Tagung der FH-DGG mit den Kolleginnen und Kollegen aus Deutschland und der Schweiz.

Steffen Birk \& Thilo Hofmann 